\title{
Carbon cycle
}

\section{The fertilization effect of $\mathrm{CO}_{2}$ on a mature forest}

\section{Yiqi Luo \& Shuli Niu}

Will mature forests absorb enough carbon from the

atmosphere to mitigate climate change as levels of carbon

dioxide increase? An experiment in a eucalyptus forest

provides fresh evidence. See p.227

When atmospheric concentrations of carbon dioxide increase, land ecosystems take up more carbon from the atmosphere as a result of increased photosynthesis, a process known as $\mathrm{CO}_{2}$ fertilization. It has long been suggested that $\mathrm{CO}_{2}$ fertilization will slow the rate of increase of $\mathrm{CO}_{2}$ levels in the atmosphere ${ }^{1}$, potentially mitigating climate change. To quantify the effect, ecologists have conducted experiments in which the atmosphere around a confined environment is enriched with $\mathrm{CO}_{2}-$ mostly in ecosystems for which the vegetation is short in stature, to reduce costs. A small number of enrichment experiments have been conducted in young forests, but there is a paucity of knowledge about the $\mathrm{CO}_{2}$-fertilization effect in mature forests. On page 227, Jiang et al. ${ }^{2}$ present results of the Free-Air $\mathrm{CO}_{2}$ Enrichment (FACE) experiment in a mature forest in Australia. Their estimate of the $\mathrm{CO}_{2}$-fertilization effect is among the lowest yet reported.

Jiang and colleagues carried out their study in a warm-temperate evergreen forest that has been undisturbed for the past 90 years, and which is dominated by eucalyptus trees (Eucalyptus tereticornis). They collected data for all the main carbon pools and fluxes in three circular plots (each 490 square metres; Fig. 1) in which the atmospheric $\mathrm{CO}_{2}$ concentration was elevated by 150 parts per million for 4 years, from 2013 to 2016 . These data were compared with those from three control plots that were not enriched in $\mathrm{CO}_{2}$.

The authors report that $\mathrm{CO}_{2}$ enrichment induced a $12 \%$ increase in carbon uptake, equivalent to an extra 247 grams of carbon per square metre per year, through gross primary production (GPP; the conversion of $\mathrm{CO}_{2}$ to organic carbon through photosynthesis). Of this, $28 \%$ ended up as net primary production (NPP; the fraction of GPP that is used for biomass growth, rather than consumed for metabolic processes) and $12.8 \%$ as an increase in the total carbon pools of the ecosystem (that is, in wood and soil). Their results add more uncertainty to already highly variable estimates of $\mathrm{CO}_{2}$ fertilization from previous $\mathrm{CO}_{2}$-enrichment experiments. How does Jiang and colleagues' estimate of the $\mathrm{CO}_{2}$-fertilization effect in this mature forest compare with results of other studies? One difference involves the leaf area of the forest canopy (the total surface area of leaves, counting only one side of the leaves), which is a major amplifier of the fertilization effect on the efficiency of carboxylation ${ }^{3}-$ the biochemical reaction that converts $\mathrm{CO}_{2}$ into organic compounds. A previous investigation ${ }^{4}$ of the same forest indicates that increased $\mathrm{CO}_{2}$ levels do not have much of an effect on the leaf-area index (LAI, a measure of total canopy leaf area) in this location, whereas $\mathrm{CO}_{2}$ enrichment did stimulate leaf-area expansion in field experiments in other ecosystems ${ }^{5,6}$.

Furthermore, the plant carbon-use efficiency - the ratio of NPP to GPP - in the

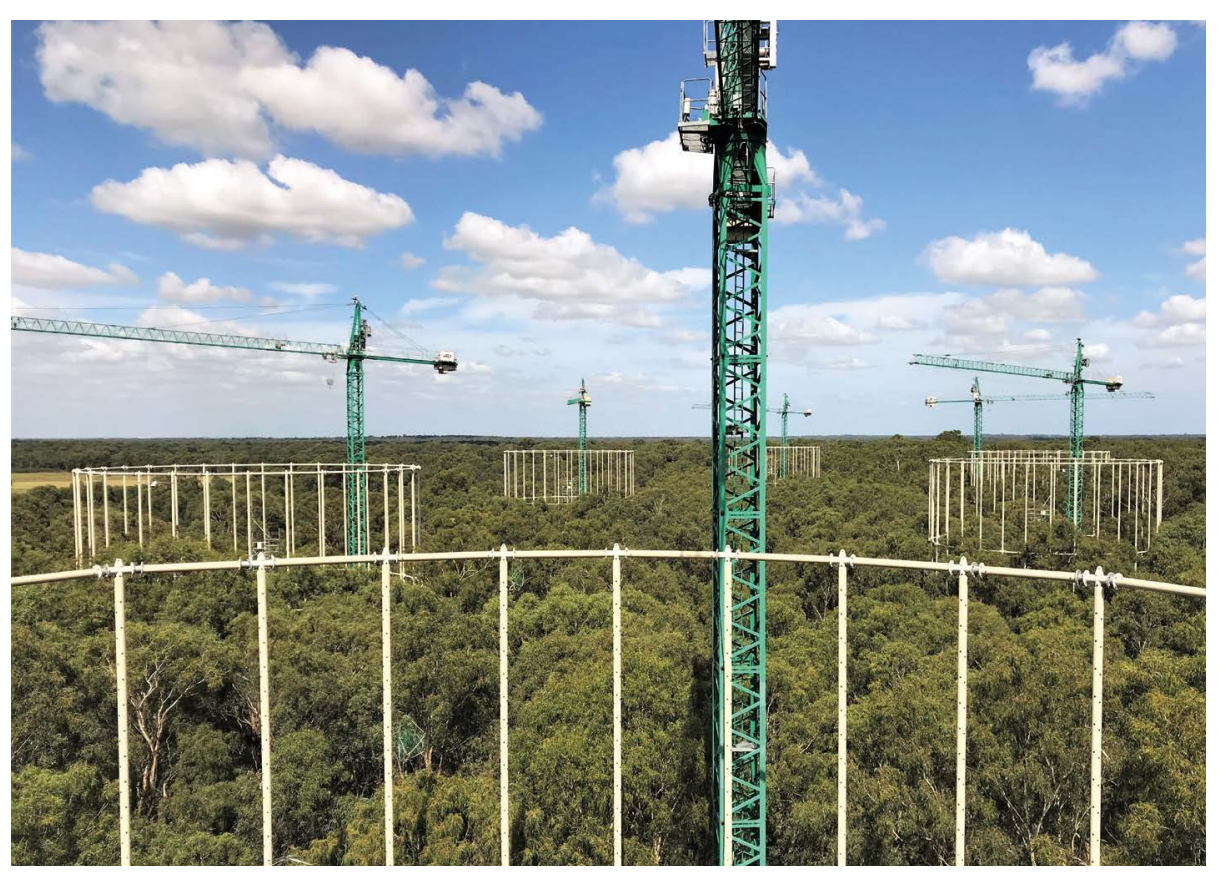

Figure 1 | Carbon dioxide enrichment in an Australian eucalyptus forest. Jiang et al. ${ }^{2}$ increased the atmospheric $\mathrm{CO}_{2}$ concentration in circular plots to observe the effects on the main carbon pools and fluxes in the forest. The cranes were used to take researchers to the top of the forest canopy, and the ring structures

transport and pump $\mathrm{CO}_{2}$ into the plots.

Australian forest, as in other mature forests ${ }^{7}$, is relatively low compared with that of young forests. This low carbon-use efficiency substantially truncates the $\mathrm{CO}_{2}$-fertilization effect. The two factors discussed above therefore jointly caused the $\mathrm{CO}_{2}$-fertilization effect in the Australian forest to be small.

How can Jiang and colleagues' results be interpreted from a more theoretical perspective? As atmospheric $\mathrm{CO}_{2}$ concentration increases, carboxylation is stimulated. This biochemical stimulation is scaled up through a biological hierarchy that progresses from leaf photosynthesis to canopy GPP, vegetation NPP, and to net changes in the carbon-pool sizes of plants and soil ${ }^{3}$. Across those scales, the carboxylation stimulation is amplified by some processes, but diminished by others.

For example, if the extra carbohydrate produced as a result of rising $\mathrm{CO}_{2}$ levels is used for leaf-area expansion to capture more $\mathrm{CO}_{2}$, then stimulation is amplified at the canopy scale (that is, through GPP). By contrast, the stimulation is diminished when the extra carbon taken up at the canopy scale is allocated for plant respiration or transferred to microorganisms for their respiration. This theoretical framework of hierarchical responses allows the fertilization effects on GPP and on other carbon-cycle processes to be approximately estimated for a scenario in which the LAI does not change much and where the $\mathrm{CO}_{2}$ concentration increases by 150 p.p.m. Indeed, Jiang and colleagues' observation-based estimates of a $12 \%$ increase in GPP, $12.8 \%$ of which ends up in the carbon pools, are very close to the lower 
limits of the theoretically derived estimates ${ }^{8}$.

Jiang and co-workers' data, and data from similar studies, can aid estimates of the global effect of $\mathrm{CO}_{2}$ fertilization. The size of this effect depends directly on the sensitivity of carboxylation efficiency to rising atmospheric $\mathrm{CO}_{2}$ levels; this sensitivity should be similar at the eucalyptus forest and at all other sites around the globe, according to a theoretical analysis ${ }^{8}$. However, as $\mathrm{CO}_{2}$ levels increase, the capacity of photosynthetic carboxylation to process more $\mathrm{CO}_{2}$ diminishes, lowering the sensitivity of carboxylation efficiency to further $\mathrm{CO}_{2}$-level increases. In other words, the $\mathrm{CO}_{2}$-fertilization effect is dwindling at the biochemical level $^{8}$. To work out the global fertilization effect, the carboxylation sensitivity is multiplied by the yearly increase in atmospheric $\mathrm{CO}_{2}$ concentration, which is becoming larger over time. The yearly increase in $\mathrm{CO}_{2}$ levels offsets the diminishing $\mathrm{CO}_{2}$-fertilization effect.

Another factor that affects the size of the global $\mathrm{CO}_{2}$-fertilization effect is the $\mathrm{LAI}^{3}$. The change in LAI observed at the Australian study site in response to $\mathrm{CO}_{2}$ enrichment is at the low end of the wide spectrum of LAI changes that have been observed elsewhere $\mathrm{e}^{4-6}$. At the global scale, however, the LAI is increasing over time - satellite observations show that Earth is literally becoming greener $^{9,10}$. The increase of LAI amplifies the $\mathrm{CO}_{2}$-fertilization effect.

The plant carbon-use efficiency reported in the current study is also at the low end of a wide range of reported values ${ }^{7}$, and contributes to the low $\mathrm{CO}_{2}$-fertilization effect observed in the study. However, we do not know much about how plant carbon-use efficiency varies over time at regional and global scales. This makes it difficult to assess whether the global fertilization effect will change because of shifts in this efficiency.

The bottom line is that it is currently difficult to estimate the size of the global $\mathrm{CO}_{2}$-fertilization effect accurately. To solve this problem, we need to know more about hierarchical constraints not only across spatial scales, from ecosystem sites to regions and the globe, but also across biological scales - from the molecular level of biochemical reactions, to the leaf and canopy scale, and through to the larger scales associated with plant production and ecosystem carbon pools.

Yiqi Luo is at the Center for Ecosystem Science and Society, Department of Biological Sciences, Northern Arizona University, Flagstaff, Arizona 86011, USA. Shuli Niu is at the Key Laboratory of Ecosystem Network Observation and Modeling, Institute of Geographic Sciences and Natural Resources Research, Chinese Academy of Sciences, and at the University of Chinese Academy of Sciences, Beijing 100101, China.

e-mail: yiqi.luo@nau.edu
1. Keeling, C. D., Chin, J. F. S. \& Whorf, T. P. Nature $\mathbf{3 8 2}$, 146-149 (1996).

2. Jiang, M. et al. Nature 580, 227-231 (2020).

3. Li, Q. et al. Biogeosciences 15, 6909-6925 (2018).

4. Duursma, R. A. et al. Glob. Change Biol. 22, 1666-1676 (2016).

5. Ferris, R., Sabatti, M., Miglietta, F., Mills, R. F. \& Taylor, G. Plant Cell Environ. 24, 305-315 (2001)

6. Uddling, J., Teclaw, R. M., Kubiske, M. E., Pregitzer, K. S. \&
Ellsworth, D. S. Tree Physiol. 28, 1231-1243 (2008)

7. DeLucia, E. H., Drake, J. E., Thomas, R. B. \& Gonzales-Meler M. Glob. Change Biol. 13, 1157-1167 (2007).

8. Luo, Y. \& Mooney, H. A. in Carbon Dioxide and Terrestrial Ecosystems (eds Koch, G. W. \& Mooney, H. A.) 381-397 (Academic, 1996).

9. Jiang, C. et al. Glob. Change Biol. 23, 4133-4146 (2017).

10. Zhu, Z. et al. Nature Clim. Change 6, 791-795 (2016).

\title{
Medical research
}

\section{Al tracks a beating heart's function over time}

\author{
Partho P. Sengupta \& Donald A. Adjeroh \\ Clinicians use ultrasound videos of heartbeats to assess \\ subtle changes in the heart's pumping function. A method \\ that uses artificial intelligence might simplify these complex \\ assessments when heartbeats are out of rhythm. See p.252
}

The heart is a specialized muscle that contracts rhythmically around its closed chambers to propel blood. However, this pumping function fluctuates throughout the day as the circulating blood flow adapts to the body's ever-changing metabolic demands ${ }^{1}$. Understanding the variations in cardiac pump activity with each heartbeat might have relevance for explaining the intricacies of heart function in health and disease. However, the tools for scrutinizing such changes remain imprecise. On page 252, Ouyang et al. ${ }^{2}$ report the development of a computational platform that uses an artificial-intelligence (AI) approach to assess cardiac ultrasound video and to provide continuous, beat-by-beat measurement of cardiac pump function.

Clinicians commonly assess cardiac function using a value termed the ejection fraction, which is the percentage of the blood volume in the left heart chamber (the left ventricle) that is pumped out when the heart contracts. In a normal heart, just over half of the blood is ejected; thus, the calculated ejection fraction is more than $50 \%$. Highly trained physicians can 'eyeball' ultrasound video loops of a beating heart and make a precise estimate of the ejection fraction ${ }^{3}$. However, if two isolated frames from the video were presented, showing only the beginning and the end of the ejection, even a trained physician would struggle to estimate the ejection fraction. Given that training and expertise vary from person to person, eyeballing is not relied on, and the ejection fraction is calculated by tracing the boundaries of the left ventricle on a digital image to estimate the blood volume at the beginning and end of ejection. It is recommended $^{4}$ that clinicians estimate the ejection fraction of a heart by tracking it over three to five heartbeats; however, in typical clinical practice, often just one beat is assessed.

If the accuracy of estimates of ejection fraction could be improved by having an easy way to routinely determine its precise value by tracking and averaging several heartbeats, this would be of immense benefit, particularly for people whose hearts are beating out of rhythm (a condition termed arrhythmia). If arrhythmia occurs, the changing duration of heartbeats alters the volume of blood filled and ejected from the left ventricle, thereby resulting in variations in the ejection fraction (Fig. 1). This variability makes the ejection fraction challenging to estimate for a type of arrhythmia known as atrial fibrillation. It is predicted ${ }^{5}$ that this condition will affect between 6 million and 12 million people in the United States by 2050 , and 17.9 million in Europe by 2060. Moreover, ejection fraction needs to be assessed frequently in people who have atrial fibrillation, because heart failure (a state characterized by a poor ability of the heart to pump blood) occurs in more than one-third of such individuals ${ }^{6}$. And more than half of people with heart failure have atrial fibrillation ${ }^{6}$.

To develop an AI-based method for assessing ejection fraction, Ouyang et al. used 10,030 cardiac ultrasound videos. These videos were stored along with images containing human-generated tracings that marked the inner border of the left ventricle at the beginning and end of the ejection cycle. The authors used a type of $\mathrm{AI}$ architecture called a convolutional neural network (CNN), first to perform a semi-automatic detection of a pattern of pixel-based information (segmentation) to recognize the left ventricle in the video frames; and second, to track the borders of the ventricle during the heartbeat 